\title{
L'interface syntaxique-sémantique de la phrase exclamative
}

\author{
Olga Kellert \\ Freie Universität Berlin \\ kellert.olga@fu-berlin.de
}

\section{Introduction}

La thèse de Obenauer (1994) sur les questions avec la particule diable (p.ex. Qui diable t'a amené jusqu'ici?) et phrases exclamatives (p.ex. quelle belle fille!) nous a montré que ces expressions sont différentes du point de vue syntaxique et sémantique des phrases interrogatives qui représentent de vraies questions. La distribution du syntagme interrogatif représente une des particularités de la phrase exclamative. L'optionalité du positionnement du syntagme qu- (anglais wh-in situ/ wh-ex situ) n'existe pas dans la phrase exclamative (et la question avec la particule diable) contrairement à la phrase interrogative:

(1) a. il aime quelle fille?

b. *incroyable, il aime quelle fille!

Une autre particularité qui n'a pas été notée par Obenauer (1994) mais qui peut être dérivée de la contrainte précédente est le fait que plusieurs syntagmes interrogatifs sont aussi exclus dans la phrase exclamative contrairement à la phrase interrogative :

(2) a. quelle fille aime quel garçon?

b. *quelle grande fille aime quel petit garçon !

Le phénomène représenté dans l'exemple (2) est décrit chez d'autres linguistes (p.ex. en catalan Castroviejo (2006), en anglais Portner \& Zanuttini, 2003, Ono, 2004, en russe Evseeva, 2006).

Un autre aspect des phrases exclamatives concerne l'interaction des quantificateurs (p.ex. chaque, plusieurs, divers, ...) et de l'opérateur qu-, c'est-à-dire que la phrase exclamative exclut l'interprétation distributive (Villalba, 2004 pour les mêmes faits en espagnol, Bacha, 2000 en français):

(3) a. ?qu'est-ce que chaque fille est lourde! (Interprétation uniforme: Les filles sont extraordinairement lourdes. Interprétation distributive: \# Marie est moins lourde que Natalie. Natalie est moins lourde que Lucia....)

b. Qu'est-ce que chaque personne pouvait voir ou entendre ? (Interprétation distributive: Mme Duvale a vu x, M. Duvale a vu y.....)

(4) a. Moi, je fais surtout du ski et du patin. Et tes copains, qu'est-ce qu'ils font comme sport? (interprétation distributive: Max fait du jogging, Pierre joue au foot,... Interprétation uniforme: \# toutes les activités sportives sont extraordinaires au même degré)

b. Oh la la ! Qu'est-ce qu'ils font comme sport, tes copains! (Interprétation uniforme: toutes les activités sportives sont extraordinaires au même degré)

La même interaction des quantificateurs a été notée indépendamment de la recherche des phrases exclamatives dans d'autres constructions $q u$-, notamment dans les phrases interrogatives qui ne représentent pas de vraies questions (Den Dikken \& Giannakidou, 2002) et dans les phrases interrogatives clivées (Baunaz, 2008 ; Guilliot, 2006) :

(5) a. Qui est-ce donc que tous les hommes aiment? (\# Paul aime Natalie, Jean aime Marie....) 
Le but de cet article est de savoir si l'on peut donner une seule explication aux contraintes syntaxiques et sémantiques notées précédemment.

La démarche sera la suivante : la deuxième section introduit quelques propositions à ce sujet. La soussection 2.1 introduit brièvement l'approche syntaxique-pragmatique de Obenauer 1994 basée sur lien discursif (D-linking) 2.2 introduit l'analyse sur la quantification (non-)distributive et sur la distribution du syntagme interrogatif de Den Dikken \& Giannakidou, 2002. La sous-section 2.3 introduit une approche adaptée à la notion d'îlots faibles de Szabolcsi \& Zwarts (1997). Contrairement aux analyses précédentes, il sera montré dans la section 3 comment la référence aux compléments des verbes présuppositionels (p.ex. regretter, savoir, prétendre, imaginer...) porte sur les faits empiriques notés précédemment. 3.1 introduit l'analyse sur la distribution du syntagme interrogatif dans les phrases exclamatives et clivées 3.2 aborde la question sur la relation entre les syntagmes interrogatifs et les quantificateurs dans les mêmes constructions.

\section{Quelques propositions d'analyse.}

\subsection{L'analyse de Obenauer (1994)}

Obenauer (1994) propose une analyse syntaxique-pragmatique de la distribution de l'élément $q u$ - dans laquelle les syntagmes interrogatifs appartenant aux constructions qui ne représentent pas de vraies questions ne sont pas liés au discours (anglais non-D-linked). Selon Obenauer (1994), l'optionalité de la distribution du syntagme interrogatif est réservée au syntagme lié au discours (anglais D-linked) (Obenauer, $1994: 526)$ ). Or, la notion de (non)-D-linked n'est pas très claire. En plus, il est difficile de dire comment le terme de non-D-linked peut être appliqué à la phrase exclamative vu qu'il est surtout associé à la particule -the hell 'diable' (Pesetsky 1987). Cependant, il y a quelques auteurs qui, dans la continuité de Obenauer 1994, après avoir analysé les syntagmes interrogatifs dans les phrases exclamatives en ont déduit qu'ils sont non-D-linked. (Wiltschko, 1997: 113), Castroviejo, 2006). Ces auteurs renvoient à la différence morpho-syntaxique entre les syntagmes interrogatifs D-linked et non-Dlinked. Ainsi, Wiltschko 1997 montre que contrairement aux phrases interrogatives le mot interrogatif ne s'accorde pas avec le syntagme nominal dans les phrases exclamatives en allemand (Wiltschko, $1997: 114)$ :

(6) Was für ein Mann!

what FÜR a man

what a man!

(7) *Welcher Mann!

which[3.Ps. Sg.m.] man

(8) Welcher Mann?

which[3.Ps. Sg.m.] man

which man?

(9) Welch ein Mann! (archaique)

which un homme

'What a man!'

De plus, le syntagme interrogatif welch 'which/what' dans l'exemple (9) dénote la forme ou la figure d'après Wiltschko 1997. Son hypothèse est renforcée par un argument diachronique. Elle suppose que le mot interrogatif welch vient du mot hwalih 'forme ou figure' en ancien allemand. Wiltschko (1997) 
présume que la dénotation de la forme ou de la figure est réservée aux expressions non-D-linked. Castroviejo (2006) propose alors une analyse similaire en y ajoutant par la suite que le syntagme interrogatif D-linked quin, comme dans quin d'aquests llibres, n'est pas accepté dans les phrases exclamatives en catalan:

(10)a. Quins llibres tan gruixuts que has llegit! 'What thick books you have read!'

b. *Quin d'aquests llibres que has llegit! 'Which one of these books you have read!'

Or, en français, on ne peut pas repérer la différence entre les syntagmes interrogatifs D-linked et non-Dlinked du point de vue morphologique:

(11)Quelle femme? (!)

Par ailleurs, il y a des raisons de proposer une analyse tout à fait contraire à celle de Obenauer (1994) qui stipulerait que le syntagme interrogatif dans la phrase exclamative peut être caractérisé comme lié au discours (D-linked) (Portner \& Zanuttini, $2000: 224)$. Les auteurs proposent une analyse des phrases exclamatives en patois de Padoue:

(12)che bel libro a to sorela, che I ghe ga regalà!

'What a nice book, to your sister, they gave her as a gift!'

Portner \& Zanuttini 2000 supposent que le syntagme interrogatif che bel libro 'what a nice book' est $D$ linked parce qu'il fait référence à un ensemble d'objet qui est présupposé: „As a D-linked element, che bel libro is presupposed to denote a salient set C of more-or-less beautiful books." (Portner \& Zanuttini, 2000 : 224). Voici un autre argument qui confirme l'hypothèse de Portner \& Zanuttini (2000). Un syntagme interrogatif $\mathrm{S}$ ne peut sémantiquement lier un pronom $\mathrm{P}$ que si $\mathrm{S}$ est $D$-linked. Voilà pourquoi les syntagmes interrogatifs non-D-linked n'apparaissent pas dans des constructions elliptiques (anglais sluicing) Lopez (2000); Merchant (2001); Den Dikken \& Giannakidou (2002):

(13)a. I know Pat wants to buy something but I don't know what he wants to buy.

b. I know Pat wants to buy something but I don't know what [e].

c. *I know Pat wants to buy something but I don't know what the hell [e]. (Lopez, 2000 : 185)

Or, le syntagme interrogatif apparait dans des constructions elliptiques des phrases exclamatives:

(14)Il change. Et avec quelle rapidité! Et avec quels dégâts! (Genestar, 2008)

La différence entre un syntagme interrogatif avec la particule -the hell 'diable' et celui d'une phrase exclamative se traduit par le fait que le premier présuppose une réponse négative tandis que le dernier présuppose toujours l'existence d'un argument:

(15) Who (the hell) would buy that book? Presupposition: Nobody would buy that book.

(16)How beautiful Marry is ! Presupposition : Marry is extremely beautiful.

Il s'ensuit que la notion de non-D-linked n'est pas très convaincante pour expliquer la distribution du syntagme interrogatif dans les phrases exclamatives.

\subsection{L'analyse de Den Dikken \& Giannakidou (2002)}

Den Dikken \& Giannakidou (2002) proposent d'analyser la contrainte sur la quantification distributive de la même façon que la contrainte sur la distribution du pronom interrogatif avec la particule -the-hell 'diable':

(17)a.what the hell did everyone buy for Max?

b. $*\left[\mathrm{C}_{\mathrm{Q}}(\ldots)\right.$ [everyone (...) [what the hell ...]]] [*everyone > what the hell, 
(> correspond à la portée large du syntagme interrogatif face au quantificateur)]

(18)a.*who is in love with who the hell?

$$
\text { b. } *\left[\mathrm{C}_{\mathrm{Q}}[\text { who (...) [who the hell] }]\right]
$$

Selon les auteurs, $\mathrm{C}_{\mathrm{Q}}$ doit $c$-commander un opérateur $q u$ - sans intervention d'un autre opérateur $q u$ - ou d'un quantificateur avec l'interprétation distributive. Les auteurs ne donnent pas de définition d'un opérateur qu-. Cependant, Rizzi (1992) nous en propose une:

(19)i. opérateur X (p.ex. qu) est un syntagme (p.ex. interrogatif) dans la position de portée (anglais scope position)

ii. position de portée est une position A-barre (anglais a left-peripheral A-bar position)

Or, lorsque deux opérateurs lient le même objet ou sont liés par le même objet, il en résulte des phrases agrammaticales:

(20)Relativized Minimality (Rizzi, 1990):

$\mathrm{X}$-governs $\mathrm{Y}$ only if there is no $\mathrm{Z}$ such that

a. $Z$ is a typical potential -governor for $Y$, and

b. Z c-commands $\mathrm{Y}$ and does not c-command $\mathrm{X}$.

Den Dikken \& Giannakidou (2002) émettent l'hypothèse que la raison pour laquelle le pronom avec la particule -the hell doit se déplacer dans la position A-barre (Spec, FocP dans leur approche) est la suivante : ce syntagme interrogatif contient un trait fort qui doit être vérifié contre Foc ${ }^{\circ}$ (Chomsky, 1995). Selon Chomsky (1995), les traits sont associés aux items lexicaux. Toutes les opérations de dérivation opèrent sur les traits syntaxiques:

(21) $\left[\mathrm{CP} \mathrm{C}_{\mathrm{Q}}\left[\mathrm{FocP}[\text { what the hell }]_{\mathrm{j}}\right.\right.$ Foc $\left.\left.\left.^{\circ}\left[\mathrm{IP} \ldots \mathrm{t}_{\mathrm{j}}\right]\right]\right]\right]$

Or, l'exemple suivant est problématique car $\mathrm{C}_{\mathrm{Q}}$ ne peut pas c-commander le syntagme interrogatif with who sans c-commander who the hell en même temps :

(22) Who the hell is in love with who?

Les auteurs supposent que le second pronom interrogatif with who 'avec qui' a une interprétation écho. Dans ce cas, ce pronom interrogatif s'adjoint au pronom interrogatif who the hell pour former une seule paire qu- (a single wh-pair) pourvu qu'un pronom écho contienne un trait $\mathrm{Q}$ à lui seul (Den Dikken \& Giannakidou, $2002: 55$ ) (La représentation suivante représente la forme logique telle qu'elle est conçue par les auteurs):

(23) $\mathrm{C}_{\mathrm{Q}}\left[[\text { Who the hell }]_{\mathrm{j}}[\text { with who }+\mathrm{Q}]_{\mathrm{k}}\right] \mathrm{t}_{\mathrm{j}}$ is in love $\mathrm{t}_{\mathrm{k}}$ ?

Selon Den Dikken \& Giannakidou (2002), le syntagme sans la particule -the-hell n'a pas besoin d'être ccommandé par $\mathrm{C}_{\mathrm{Q}}$ puisqu'il contient déjà un trait $\mathrm{Q}$ à lui seul.

Mis à part le fait que l'adjonction à droite n'est pas possible dans le cadre génératif proposé par Kayne (1994) et que la proposition du mouvement de l'opérateur qu- sur la forme logique a été critiquée par beaucoup de linguistes (Reinhart, 1998 entre autres), on peut se demander pourquoi l'interprétation écho n'est pas possible dans (18a). Finalement, la question qui se pose alors est: qu'est ce qu'ont en commun les phrases exclamatives et autres constructions similaires avec les constructions wh-the-hell?

La section suivante propose une analyse similaire qui n'a pas recourt à la notion de traits de Chomsky (1995). 


\subsection{L'analyse adaptée à Szabolcsi \& Zwarts (1997)}

Szabolcsi \& Zwarts (1997) s'occupent d'îlots faibles, ou sélectifs, qui autorisent certains mouvements et en bloquent d'autres. Contrairement aux analyses syntaxiques (Rizzi, 1990 ; Cinque 1990), Szabolcsi \& Zwarts (1997) proposent une analyse sémantique d'îlots faibles. Selon les auteurs, seuls les syntagmes interrogatifs qui ne sont pas associés à une liste d'éléments discrets sont susceptibles aux effets d'îlots (bad extractees). Ces syntagmes sont typiquement associés à la notion de manière, raison et degré. Or, la négation représente un îlot faible dans le système de Szabolcsi \& Zwarts (1997) car la négation dénote l'opération de complémantation ${ }^{1}$ qui exclut une dénotation definie de certains syntagmes interrogatifs. Voyons quelques exemples:

$$
\begin{aligned}
& \text { (24)a. *Quel âge n'as-tu pas? } \\
& \text { b. Quel jeu n'as-tu pas encore acheté? }
\end{aligned}
$$

La question (24a) présuppose l'existence d'un argument exprimé par le syntagme interrogatif afin d'être interprétée par l'interlocuteur. Or, le complémentaire de l'extension du prédicat ' $\lambda \mathrm{n}$ (tu a n ans)' n'est pas défini. Voilà pourquoi l'interlocuteur ne peut pas interpréter la question. En revanche, le complémentaire de l'extension $d u$ prédicat ' $\lambda x$ (tu a acheté $x$ )' peut être défini car la question en (b) présuppose l'existence d'un jeu $x$ tel que tu n'as pas acheté ce jeu. En résumé, la question (24a) non est définie car le syntagme interrogatif ne constitue pas un domaine bien défini de sorte que l'interlocuteur puisse choisir une valeur appropriée de cette liste.

Avant de montrer comment l'approche de Szabolcsi \& Zwarts (1997) pourrait expliquer quelques faits empiriques, il faut introduire l'interprétation du syntagme interrogatif dans les phrases exclamatives. La phrase exclamative dans (25) et la phrase déclarative avec l'adverbe même dans (26) ont une interprétation similaire:

(25)Qu'est-ce que Marie est grande!

(26) Marie aime même Pierre.

Voici les implications qui sont suggérées par ces phrases.

a. Le locuteur ne s'attendait pas à ce que Marie soit grande de degré x. (Présupposition ou implication conventionnelle)

a' Le locuteur ne s'attendait pas à ce que Marie aime Pierre.

Bref, l'implication dans (a et a') exprime une violation de l'attente (violation of expectation):

La phrase exclamative - toute phrase exclamative - prend appui sur l'évidence : $p$ est asserté avec force dans $\mathrm{m}_{0}$. Mais en même temps est suggérée la fausseté de $\mathrm{p}$ dans quelque monde contrefactuel: cette fausseté relève d'une image d'univers, contradictoire avec mon univers actuel. (Martin, 1987:98).

b' $\forall x(x \neq$ grande au degré $x+1)$ :le locuteur s'attendait plus à ce que Marie soit grande de degré $\mathrm{x}$ qu'elle soit grande de degré $\mathrm{x}+1$. (Implication d'une échelle, anglais scalar implicature)

b'. $\forall x(x \neq$ Pierre): le locuteur s'attendait plus que Marie aime $x$ qu'elle aime Pierre.

c. Il y a un degré $\mathrm{x}(\mathrm{x} \neq \mathrm{x}+1)$ du prédicat grand tel que Marie est grande au degré $\mathrm{x}$. (présupposition existentielle)

c'. Il y a un $\mathrm{x}$ ( $\mathrm{x} \neq$ Pierre) tel que Marie aime $\mathrm{x}$.

Il existe un débat à propos de savoir si la valeur associée par le syntagme interrogatif dans les phrases exclamatives représente une valeur maximale sur une échelle de degré (Portner \& Zanuttini, 2003) ou si cette valeur est juste la plus haute des valeurs alternatives présupposées (Michaelis, 2001). Selon Portner \& Zanuttini, 2003 l'implication de la valeur maximale du degré se traduit par l'impossibilité de sa négation: 
(27)Qu'est-ce qu'elle est grande (pour une fille de 10 ans)!

a.\#Je ne sais pas si elle est grande du tout.

b.\#Elle est grande mais elle n'est pas très grande pour une fille de 10 ans.

Or, l'implication de degré et d'une valeur maximale prédestine le syntagme interrogatif à un élément qui ne peut pas être extrait de la portée sémantique (anglais scope) d'un autre opérateur Opx. (p.ex. opérateur de complémentation). Il s'ensuit que le syntagme interrogatif ne peut pas contenir d'autres opérateurs, Opx, dans sa portée sémantique (représentée par $>$ ):

$(28) *$ Qu'est-ce que $_{j}>$ Opx.> DegréP ${ }_{j}$ !

Un exemple de cette contrainte est l'intervention de la négation:

(29)*Comme/(Qu'est-)Ce que Marie n'est pas belle!

Il en va de même avec les quantificateurs universels et d'autres syntagmes interrogatifs ayant un rapport à l'échelle de degré:

(30)a. *quelle grande fille aime quel petit garçon!

b. ?qu'est-ce que chaque fille est lourde! (\# Marie pèse $90 \mathrm{~kg}$, Natalie $80 \mathrm{~kg} . .$. )

c. Oh la la ! Qu'est-ce qu'ils font comme sport, tes copains! (\# interprétation distributive)

L'analyse proposée ressemble beaucoup à celle de Den Dikken \& Giannakidou (2002). Cependant, elle n'est pas basée sur la notion des traits forts (Chomsky, 1995). Par contre, elle est basée sur la notion de degré afin d'expliquer les effets d'intervention de quelques opérateurs dans les phrases exclamatives.

Or, cette analyse n'explique pas la contrainte de wh-in situ dans les phrases exclamatives (cf. 1b) et dans d'autres constructions emphatiques (p.ex. questions rhétoriques, phrases interrogatives clivées):

(31)a. *Vous êtes qui diable, pour parler ainsi?

b. Qui diable êtes vous? (Obenauer, 1994:338)

(32)a. C'est qui qui a mangé le fromage?

b. Qui a mangé quoi

c. ${ }^{*}$ ''est qui qui a mangé quoi? (Lambrecht, 1996:330)

Or, les pronoms interrogatifs de ces constructions (p.ex. qui, quoi) sont associés aux éléments discrets et constituent alors un domaine bien défini. La notion de bad extractees ne tient pas compte des exemples précédents. Il faut noter qu'on pourrait expliquer l'exemple (31a) en tenant compte de l'aspect sociolinguistique (cf. Zribi-Hertz 2006:14), puis que l'optionalité de la distribution du syntagme interrogatif n'existe que dans le Français parlé. Or, la particule diable n'y est plus utilisée. Cependant, cette explication est très limitée, car les faits $(1,2)$ ainsi que (32) restent inexpliqués.

La section suivante propose une analyse selon laquelle le caractère présuppositionel des phrases emphatiques (des phrases exclamatives et des phrases clivées) est responsable de la contrainte de la distribution du syntagme interrogatif.

\section{Le rôle de la présupposition dans les constructions emphatiques}

En ce qui concerne la notion de présupposition sémantique (définition vériconditionnelle), elle est definie de la manière suivante: "Une proposition $p$ présuppose une proposition $q$ si $q$ doit être vraie pour que $p$ ait une valeur de vérité, quelle qu'elle soit. Le test classique de la négation : la présupposition d'une phrase est ce qui de cette phrase survit à l'epreuve da sa négation." (Latraverse, 1987 :151). Voici ses exemples:

(33)a. Tous les enfants de Jean sont chauves. Présupposition : Jean a des enfants. 
b. Tous les enfants de Jean ne sont pas chauves. Présupposition : Jean a des enfants.

La présupposition vériconditionelle s'exprime aussi dans le cas des verbes factifs. L'emploi de tels verbes présuppose que leur complément est vrai:

(34)Max sait que Chirac a perdu les élections. Présupposition : Chirac a perdu les élections.

Or, il existe aussi une notion pragmatique de la présupposition comme condition d'emploi. Selon Stalnaker (1977) la présupposition pragmatique est liée à l'ensemble de croyances d'arrière-plan aux énonciations du locuteur (anglais common ground) (Moeschler \& Reboul, 1994). S'il n'y a pas de base de croyances partagées entre le locuteur et son interlocuteur, la communication n'est pas possible. Il en découle alors que ce sont des locuteurs qui ont des présuppositions et non des énoncés (Moeschler \& Reboul, 1994). Dans l'exemple suivant c'est le locuteur qui présuppose fortement que Chirac a perdu les élections. (Moeschler \& Reboul, 1994):

(35)Max fut surpris que Chirac perde les élections.

Les sous-sections suivantes montrent si et comment la présupposition est liée aux contraintes dans les phrases emphatiques (p.ex. phrases exclamatives).

\subsection{La contrainte sur la distribution du syntagme interrogatif}

Observons les exemples suivants:

(36)a. C'est à qui que Marie l'a donné? => Il y a quelqu'un à qui Marie l'a donné. (Présupposition existentielle)

b. Est-ce que Marie l'a donné à quelqu'un? $\neq>$ Il y a quelqu'un à qui Marie l'a donné. (\#Présupposition existentielle)

(37)a. Quel homme l'a épousée! => Il y a quelqu'un qui l'a épousée.

b. Est-ce que quelqu'un l'a épousée? $\neq>$ Il y a quelqu'un qui l'a épousée.

(38)a. Qui alors peut le faire (si ce n'est pas Pierre)? => Personne, sauf Pierre, ne peut le faire.

b. Qui peut le faire ? $\neq>$ Personne ne peut le faire.

On peut observer que toutes les constructions en (a) montrent la contrainte de la distribution du syntagme interrogatif:

(39)a. *Qui alors peut faire quoi (si ce n'est pas Pierre)?

b. ${ }^{*}$ 'est quoi que Pierre a donné à qui ?

c. *Quel homme a épousé quelle femme !

La construction exclamative dans (37a) et la construction rhétorique (38 a) présupposent une réponse ou une proposition par le locuteur. Leur caractère présuppositionel est évident, puisque ces constructions ne sont pas utilisées comme vraies questions en dépit de leur forme interrogative. Or, la phrase interrogative clivée, en (36a), est une vraie question. Cependant, elle peut être classée dans la même catégorie des formes interrogatives présuppositionnelles, car le locuteur présuppose l'existence de la valeur associée au pronom interrogatif, il ne cherche qu'à l'identifier. En revanche, une question non-clivée n'est pas nécessairement présuppositionnelle (Chang, 1997). Comparons une question non-clivée (40) avec une question clivée (41):

(40)A : Qu'est-ce qu'il a donné à Paul? / Qu'a-t-il donné à Paul ? B : Rien.

A : Ah bon? Il ne lui a rien donné?

(41)A : C'est quoi (ce) que Pierre lui a donné ? B ?? Rien. 


\section{A : Ah bon ? ${ }^{? ?}$ C'est rien qu'il lui a donné?}

Vu l'aspect présuppositionel des phrases clivées, exclamatives et rhétoriques, je propose d'analyser ces constructions en termes d'opérateur présuppositionnel qui prend une phrase comme complément représentant une proposition présupposée par le locuteur. Il est présumé qu'une proposition présupposée correspond à une description définie (Honcoop, 1998). Une description définie n'introduit jamais de nouveaux référents de discours (anglais new discourse referents) et elle n'a aucune influence sur l'ensemble de connaissances. Or, un nouveau référent est souvent introduit par une expression indéfinie ou un syntagme interrogatif. Par conséquent, une proposition présupposée ne peut jamais contenir un pronom indéfini ou un syntagme interrogatif dépendant d'un antécédent hors de sa portée. Or, la proposition présupposée par le locuteur est exprimée linquistiquement par une phrase subordonnée ou relative, $\mathrm{CP}_{\text {sub/rel }}$, dans la construction clivée et par une phrase temporelle, TP, dans la construction exclamative:

(42)a. [CP Quelle belle femme ${ }_{j}\left[\mathrm{TP}\right.$ il a épousée $\left.\left.t_{j}\right]\right]$ !

b. [TP C'est quoi ${ }_{j}\left[\mathrm{CP}_{\text {subrel }}\right.$ que tu lui as donné $\left.\left.\mathrm{t}_{\mathrm{j}}\right]\right]$ ?

Voyons alors, quelles sont les configurations possibles de la relation entre l'opérateur présuppositionnel (représenté par $O p_{\text {pré }}$ ) et un pronom indéfini ou interrogatif (ZP) dépendant d'un (autre) antécédent (Opx):

(43)a. ${ }^{*} \mathrm{Opx}_{\mathrm{j}}>\mathrm{Op} \cdot \mathrm{prere}>\mathrm{ZP}$

$\left.\mathrm{b} *\left[\mathrm{Opx}_{\mathrm{j}}\left[\mathrm{Op} \mathrm{pré}_{\text {é }} \text { Elle a [quelles belles jambes }\right]_{\mathrm{j}}\right]\right]$ !

c. *[Opx ${ }_{j}$ Quelle belle femme $\left.\left.{ }_{j}\left[O p_{\text {pré }} \text { a épousé [quel homme riche }\right]_{j}\right]\right]$ !

d. *[Opx ${ }_{\mathrm{j}} \mathrm{C}$ 'est quoi $\left.\left.{ }_{\mathrm{j}}\left[\mathrm{Op} \mathrm{pré}_{\mathrm{e}} \text { que tu as donné [à qui }\right]_{\mathrm{j}}\right]\right]$ ?

(44) a. $\mathrm{Opx}_{\mathrm{j}}>\mathrm{ZP}_{\mathrm{j}} \mathrm{Op} \cdot \mathrm{pré}>\mathrm{t}_{\mathrm{j}}$

b. $\left[\mathrm{Opx}_{\mathrm{j}}\right.$ Quelle belle femme $\mathrm{j}\left[\mathrm{Op} \mathrm{p}_{\text {pré }}\right.$ il a épousée $\left.\left.t_{\mathrm{j}}\right]\right]$ !

c. $\left[\mathrm{Opx}_{\mathrm{j}} \mathrm{C}\right.$ 'est quoi $\mathrm{i}_{\mathrm{j}}\left[\mathrm{Op}_{\text {pré }}\right.$ que tu lui as donné $\left.\mathrm{t}_{\mathrm{j}}\right]$ ?

Il est très important de savoir à quel niveau une configuration comme (43a) s'applique du point de vue d'une grammaire modulaire (selon Chomsky, 1995). Vu la différence entre (43) et (44), la configuration dans (44) s'applique après le mouvement de ZP.

Le rôle de l'opérateur présuppositionnel comme projecteur d'îlot est bien connu dans la littérature (Honcoop, 1998, Mathieu \& Butler, 2004):

$(45){ }^{*}$ Combien $_{j}$ regrettes-tu qu'il ait mangé $t_{j}$ de pommes?

(46)?Combien ${ }_{j}$ penses-tu qu'il a mangé $t_{j}$ de pommes ?

Il est supposé que le quantificateur combien doit lier son argument - l'élément indéfini de pommes. Un tel lien est soumis à certaines contraintes relatives aux îlots effectués par l'opérateur présuppositionnel (Honcoop, 1998 ; Mathieu \& Butler, 2004). Rapellons que le caractère présuppositionel peut être exprimé par le complément des verbes factifs comme regretter dans (45). Il se trouve que la même configuration (43a) est valide pour (45).

En résumé, il a été montré qu'il y a une corrélation entre le caractère présuppositionel d'une phrase et le fait que cette phrase ne peut pas contenir un pronom indéfini ou interrogatif dans son domaine. Cette corrélation semble être due à une contrainte pragmatique. 


\subsection{Les effets des quantificateurs dans les phrases exclamatives.}

Bacha $(2000: 56)$ constate que les phrases exclamatives sont peu compatibles avec des pronoms indéfinis et/ou des quantificateurs distributifs en fonction de sujet. Son observation est confirmée par Villalba (2004).

(47)a.??Qu'est-ce que divers étudiants se sont trompés!

b.Qu'est-ce qu'ils se sont trompés!

(48)a.??Qu'est-ce que plusieurs enfants crient!

b.Qu'est-ce que ses enfants crient!

c.??Qu'est-ce que chaque enfant crie!

En principe, les quantificateurs comme divers, plusieurs et chaque provoquent une interprétation distributive s'ils sont appliqués à un pronom indéfini ou un pronom interrogatif. Or, l'interprétation distributive paraît incompatible avec la phrase exclamative selon le propre jugement de Bacha (2000) et de Villalba (2004).

L'argumentation suivante a pour but de montrer la relation entre une réponse exhaustive et l'absence de quantification distributive dans les phrases exclamatives et autres formes emphatiques.

La quantification distributive donne l'interpétation d'une quantification sur des paires (p.ex. il y a plusieurs paires de garçon-fille telles que le premier aime le second):

(49)(a) Chaque garçon aime une fille.

(b) $(\forall x)$ [ garçon $(x)=>($ il y un $x)$ [ fille $(y) \&$ aime $(x, y)]]$

(c) Pour tout $\mathrm{x}$, si $\mathrm{x}$ est un garçon, alors il $\mathrm{y}$ a une fille $\mathrm{y}$ telle que $\mathrm{x}$ aime $\mathrm{y}$.

(50)(a) Chaque garçon aime qui?

(b) $(\forall x)$ [ garçon $(x)=>$ (il y un $x)$ [humain $(y) \&$ aime $(x, y)]]$

(c) Pour tout $\mathrm{x}$, si $\mathrm{x}$ est un garçon, alors il $\mathrm{y}$ a quelqu'un $\mathrm{y}$ telle que $\mathrm{x}$ aime $\mathrm{y}$.

Or, dans un contexte spécifique, la quantification distributive peut donner une quantification sur une seule paire:

(51)Chaque garçon aime une (seule) fille.

Proposition exhaustive: Chaque garçon aime Marie.

(52) Chaque garçon aime QUI?

Réponse exhaustive: Chaque garçon aime Marie.

Notons que des questions avec plusieurs pronoms ou syntagmes interrogatifs peuvent aussi avoir une réponse exhaustive ou non-exhaustive (Corblin \& Tovena 2003):

(53)Qui aime qui? Réponse non-exhaustive (p.ex. Marie aime Pierre, Paul aime Jeanne, etc.)

Un contexte spécifique peut donner une réponse exhaustive :

(54)A : Marie a tué Pierre. B : Hein ? Qui a tué qui?

Il existe cependant quelques constructions interrogatives qui présupposent une réponse exhaustive. La phrase clivée, la phrase rhétorique et la phrase interrogative dont le pronom interrogatif est fortement accentué, présupposent en principe une seule réponse:

(55)Qui est-ce donc que tous les hommes (de ton travail) aiment?

\# Paul aime Natalie, Jean aime Marie, etc.

(56)A: Chaque fille (de ma classe) aime un garçon. B: Ah oui. Qui c'est déjà?

A: \# Marie aime Paul, Pauline aime Jean, etc. 
(57)A : Chaque fille (de ma classe) a acheté un cadeau. B : Ah bon ? Et c'est quoi ce cadeau ? \# Marie a acheté $\mathrm{x}$, Pauline a acheté $\mathrm{y}$, etc.

(58)Quand est ce que chaque citoyen va prendre ses responsabilités ? réponse exhaustive, \# réponse non-exhaustive

(59)Quoi de plus dérangeant pour chaque interlocuteur (que d'entendre du bruit de fond ou des voix parasites pendant les conversations)? Réponse exhaustive.

L'interprétation exhaustive est d'autant plus visible dans les phrases clivées qui excluent tous les adverbes ayant une interprétation non-exhaustive :

(60)A: C'est à qui que tu as parlé à la soirée de Bertrand?

B: \#(J'ai parlé) à Marie, entre autres / par exemple. (Shlonsky 2008:5)

Il est très important de noter qu'une réponse exhaustive n'exclut pas une réponse fonctionnelle (Kiss, 1991 ; Chierchia, 1993):

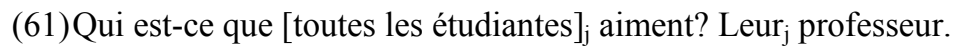

L'exemple (61) montre qu'une réponse fonctionnelle implique logiquement une quantification distributive, sauf que la distribution est uniforme, c'est-à-dire il se trouve que par hasard toutes les paires (étudiante-qui) sont uniformes (étudiante-prof) (Rebuschi, 1994).

Rappelons que Den Dikken \& Giannakidou (2002) proposent de représenter la réponse exhaustive dans les constructions interrogatives avec la particule -the-hell 'diable' en supposant qu'il y a une symétrie entre la portée large du pronom interrogatif (wide scope) face au quantifacateur distributif et la position structurelle de ces éléments: le pronom interrogatif c-commande le quantificateur en cas de portée large. Selon les auteurs, une portée étroite du pronom interrogatif (narrow scope) correspond à la représentation selon laquelle le quantificateur distributif everyone 'chacun' c-commande le pronom interrogatif. Dans ce cas, le complémentateur illocutoire $\mathrm{C}_{\mathrm{Q}}$ qui doit lier le syntagme interrogatif pour des raisons interprétatives ne peut plus le c-commander sans l'intervention du quantificateur distributif everyone 'chacun':

(62)a.what the hell did everyone buy for Max?

( $>$ correspond à la portée large du syntagme interrogatif face au quantificateur)

b. $*\left[\mathrm{C}_{\mathrm{Q}}(\ldots)\right.$ [everyone (...) [what the hell ...]]] [*everyone 'chacun'> what the hell 'que diable']

Cependant, ce propos ne peut pas expliquer l'exemple (63) qui présuppose à la fois une réponse exhaustive et la reconstruction syntaxique de la portée étroite du pronom interrogatif due à la réponse fonctionnelle (Rebuschi, 1994 : 126):

(63) chaque fille aime quelqu'un (= sa propre mère)

Rapellons aussi que l'hypothèse d'une reconstruction syntaxique de la portée doit faire la différence entre une réponse exhaustive de type (63) et (64):

(64)toutes les filles aiment quelqu'un (une seule mère, p.ex. celle de Marie)

Revenons sur la quantification de la phrase exclamative. L'exemple (65) montre que la phrase exclamative exclut une interprétation non-uniforme ou distributive, tandis qu'une phrase interrogative ne l'exclut pas dans l'exemple (66) :

(65)Quelle belle image ils veulent donner d'eux! (interprétation uniforme: ils veulent tous donner une très belle image, interprétation non uniforme : \# ils veulent donner une image différente)

(66)Quelle image veulent-ils donner d'eux? (interprétation non uniforme : ils veulent donner une image différente) 
Or, l'interprétation uniforme d'une phrase exclamative reste irréprochable en dépit de la reconstruction d'un syntagme nominal dans une position structurelle qui est c-commandée par le sujet pronominal ils (Rapellons que le pronom ils qui est l'antécédent de l'anaphore image d'eux mêmes doit c-commander l'anaphore selon la condition A de la théorie du Liage de Chomsky (1986)):

(67) [ст Quelle belle image d'eux -mêmes [IP ils veulent donner quelle belle image d'eux-mêmes]]!

Il s'ensuit que la contrainte (43a) répétée dans (68) ne s'applique pas sur la représentation de la reconstruction structurelle de l'interprétation uniforme:

$(68) * \mathrm{Opx}_{\mathrm{j}}>\mathrm{Op}_{\cdot p r e ́}>\mathrm{ZP}_{\mathrm{j}}$

(69) [ CP $_{\text {Opx }} \mathrm{Op}_{\mathrm{j}}$ Quelle belle image d'eux - mêmes [IP $_{\mathrm{j}} \mathrm{Op}_{\text {.pré }}$ ils veulent donner quelle belle image d'euxmêmes $\mathrm{j}]$ !!

La réponse à la question principale est donc négative. La contrainte sur la distribution du syntagme interrogatif et la contrainte sur la quantification ils reçoivent une explication différente contrairement au propos de Den Dikken \& Giannakidou (2002).

\section{Recherches futures}

Il est important de noter que notre propos ne dit rien sur l'interprétation du pronom interrogatif qui est soumis sous la portée d'un opérateur (Opx) dans (68). L'exploration détaillée de cet opérateur qui lie le pronom interrogatif est réservée à de futures recherches. Néanmoins, on pourrait supposer selon Rullmann 1995 que le pronom qu- se réfère à un élément maximal de l'ensemble des éléments dénotés par le pronom interrogatif (Rullmann, 1995 ; Matushansky \& Ruys, 2006). Il se trouve ainsi que l'élément qu- des phrases exclamatives est soumis à un opérateur de maximalité. L'opérateur de maximalité est utilisé afin de sélectionner le plus grand degré au sein de l'ensemble des degrés associés à un individu (Matushansky \& Ruys, 2006). Rappelons que le syntagme interrogatif dans la phrase exclamative exprime une valeur maximale de degré comme cela a été montré dans l'exemple (27).

Il semble problématique d'analyser les phrases interrogatives clivées et d'autres constructions emphatiques (p.ex. questions avec la particule the-hell) de la même manière que les phrases exclamatives, c'est-à-dire en termes de maximalisation. Cependant, une étude plus détaillée pourrait montrer que cette impression est fausse.

Se pose ensuite la question de savoir pourquoi le pronom interrogatif qui mais pas quelle (AP) NP est accepté plus d'une fois :

(70)Incroyable, qui aime qui! réponse non-exhaustive, réponse exhaustive

(71)Incroyable, *quel petit homme aime quelle grande femme !

Il est important de noter que l'exemple 70) peut avoir une interprétation non-exhaustive ou bien distributive-non uniforme. On pourrait supposer que le pronom interrogatif qui est associé aux individus résiste à l'effet des îlots, tandis que le syntagme interrogatif quel $A P N P$, associé aux degrés, ne le fait pas (Szabolcsi \& Zwarts, 1997).

Un autre aspect problématique nous provient du japonais. Selon Ono (2004), le japonais n'exclut pas la possibilité de plusieurs mots $q u$ - dans une phrase exclamative qui contient deux syntagmes nominaux interrogatifs :

(72)a.*What a long paper John sent to what a famous journal!

b.John-wa nante nagai ronbun-o nante yuumeina zassi-ni oku-tta no da J-TOP wh long paper-ACC wh famous journal-DAT send-PST NL.COP

'What a long paper John sent to what a famous publisher!' (Ono, $2004: 1)$ 
L'exemple grammatical en japonais pose problème pour donner une explication qui est censée être universelle. Dans tous les cas, il est trop tôt pour dire si l'explication de la contrainte de plusieurs mots $q u$ - dans une phrase exclamative et/ou rhétorique est une contrainte universelle.

On pourrait supposer qu'un seul opérateur illocutoire lient deux variables en japonais:

$$
\text { (73)Op. } \mathrm{x}_{\mathrm{j}} \ldots \mathrm{ZP} 1_{\mathrm{j}} \ldots \mathrm{ZP} 2_{\mathrm{j}} \text {. }
$$

L'exploration des conditions du liage de deux expressions par un opérateur est réservée à nos recherches futures.

Il semble qu'il y ait des variations en ce qui concerne la distribution de plusieurs syntagmes interrogatifs dans une phrase et que cette possibilité peut varier d'une langue à l'autre (japonais contre français), d'une construction à l'autre (question informative contre construction exclamative), d'un syntagme interrogatif à l'autre (qui contre quel $A P N P$ ). L'exploration approfondie et détaillée de la façon dont ces variations doivent être formalisées sera réservée à nos recherches futures.

\section{Références bibliographiques}

Bacha, J. (2000). L'exclamation. Paris : L’Harmattan.

Baunaz, L. (2008). Non-canonical quantification: a syntactic approach to French quantification. Ph.D dissertation. University of Geneva.

Butler, A. \& Mathieu, E. (2004). The Syntax and Semantics of Split Constructions: A Comparative Study. Basingstoke/New-York : Palgrave-MacMillan.

Castroviejo, E. (2006). Wh-Exclamatives in Catalan. Universitat de Barcelona, Doctoral dissertation.

Chang, Lisa (1997). Wh-in-situ phenomena in French. Master's thesis, University of British Columbia.

Chierchia, G. (1993). Questions with Quantifiers. Natural Language Semantics, 1, 181-234.

Chomsky, N. (1986) Knowledge of Language. Dordrecht : Foris.

Chomsky, N. (1995) The Minimalist Program. (Current Studies in Linguistics 28.), Cambridge, Mass : MIT Press.

Cinque, G. (1990). Types of f-Dependencies. Cambridge, Mass : MIT Press.

Corblin, F. \& Tovena, L. M. (2003). L'expression de la négation dans les langues romanes in D. Godard ed. Les langues romanes: problèmes de la phrase simple, Paris : CNRS Editions, 281-343

Den Dikken, M. \& Giannakidou, A. (2002). From hell to polarity. Linguistic Inquiry, 33 (1), 31-61.

Evseeva, E. (2006). On the distribution and interpretation of Wh-elements in Russian as contrasted with English and Japanese. Ms. [http://hdl.handle.net/2433/57304.pdf]. December 2007.

Guilliot.N. (2006) La reconstruction à l'interface entre syntaxe et sémantique. Thèse de doctorat, Université de Nantes.

Honcoop, M. (1998) Dynamic excursions on weak islands. Doctoral dissertation, Leiden University. The Hague: Holland Academic Graphics.

Kayne, R.S. (1994). The Antisymmetry of Syntax. Cambridge: MIT press.

E. Kiss, K. (1991) Logical Structure in Syntactic Structure. C.-T. J. Huang and R. May (eds.), Logical Structure and Linguistic Structure, Dordrecht : Kluwer, 111-147.

Krifka, M. (1991). A compositional semantics for multiple focus constructions. S. Moore \& A.Z. Wyner (Eds.), Proceedings of the First Semantics and Linguistic Theory Conference (SALT 1). Cornell University.

Latraverse, F. (1987) La pragmatique: histoire et critique. Bruxelles : Mardaga.

Martin, R. (1987) Langage et Croyance. Bruxelles : Mardaga. 
Matushansky, O. \& E.G. Ruys (2006) Meilleurs voeux: Quelques notes sur la comparaison plurielle. Empirical Issues in Syntax and Semantics; 6, O. Bonami \& P. Cabredo Hofherr (eds.), 309-330.

Michaelis L. (2001). Exclamative constructions. Language Typology and Language Universals, vol. 2. Berlin : de Gruyter.

Moeschler, J. \& A. Reboul (1994) Dictionnaire encyclopédique de pragmatique, Paris : Seuil.

Obenauer, H.-G. (1994). Aspects de la syntaxe A-barre: (...). Paris : Thèse de doctorat, Université Paris 8.

Ono, H. (2004). On Multiple Exclamatives. Presented at Georgetown University Roundtable in March 2004. [http://www.ling.umd.edu/events/StudentConference/ono04.pdf.]

Pesetsky, D. (1987). Wh-in-situ: movement and unselective binding. In : Reuland, E. and Meulen, A., (eds), The Representation of (In)definiteness. Cambridge Mass : MIT Press, 98-129.

Portner, P. \& Zanuttini, R. (2003). Exclamative clauses: At the syntaxsemantics interface. Language, 79 (1), 39-81.

Portner, P. \& Zanuttini, R. (2000). The force of negation in Wh exclamatives and interrogatives. In L. Horn and Y. Kato (Eds.), Studies in Negation and Polarity: Syntactic and Semantic Perspectives, 201-39. New York and Oxford: Oxford University Press.

Rebuschi, G. (1994) Skolémisation et (non-)spécificité. Faits de langues, 4, 121-128.

Reinhart, T. (1998) Wh-in-situ in the framework of the minimalist program. Natural Language Semantics, 6, 29-56.

Rizzi, L. (1990). Relativized Minimality. Cambridge Mass.

Rizzi, L. (1992). Argument/adjunct (a)symmetries. Northeast Linguistics Society, 22, 365-381.

Rullman, H. (1995). Maximality in the Semantics of Wh-Constructions, Doctoral dissertation, University of Massachusetts, Amherst.

Shlonsky, U. (2008). A talk in four unequal parts. Talk held at the University of Siena. available at [http://www.ciscl.unisi.it/doc/doc_ev/shlonsky08-talk.pdf]. May 2008.

Spector, B. (2006). Aspects de la pragmatique des opérateurs logiques. Ph. D. thesis, Université Paris 7.

Szabolcsi, A. \& F. Zwarts (1997) Weak islands and an algebraic semantics of scope taking. Ways of Scope Taking, edited by A. Szabolcsi, 217-262. Dordrecht: Kluwer.

Villalba, X. (2004). Exclamatives and negation. Research report, Grup de Gramatica Teorica, Universitat Autònoma de Barcelona (http://seneca.uab.es).

Wiltschko M. (1997) D-linking, scrambling and superiority in German. Groninger Arbeiten zur Germanischen Linguistik 41, 107-142.

${ }^{1}$ «Soit D le domaine du modèle dans lequel on évalue une phrase, c'est-à-dire le domaine des objets qui peuvent servir de valeur pour les variables. Soit E un sous-ensemble de D. Le complémentaire de E est l'ensemble E' qui contient tous les individus de D qui n'appartiennent pas à E. » (Spector, 2006, chap. 4). 